\title{
Constraint on the early expansion of XRF 080109, related to supernova SN 2008D
}

\section{Zsolt Paragi*}

JIVE, Dwingeloo, The Netherlands

MTA Research Group for Physical Geodesy and Geodynamics, Penc, Hungary

E-mail: zparagi@jive.nl

\section{Alexander J. van der Horst}

NASA Postdoctoral Program Fellow, NASA/MSFC/ORAU, Huntsville, AL, USA

E-mail: alexander.j.vanderhorst@nasa.gov

\section{Chryssa Kouveliotou}

NASA/MSFC, Huntsville, AL, USA

E-mail: chryssa.kouveliotou@nasa.gov

\section{Michael A. Garrett}

Astron, Dwingeloo, The Netherlands

University of Leiden, The Netherlands

E-mail: garretteastron.nl

\section{Ralph A.M.J. Wijers}

University of Amsterdam, The Netherlands

E-mail: r.a.m.j.wijers@uva.nl

\section{Jonathan Granot}

University of Hertfordshire, UK

E-mail: j.granot@herts.ac.uk

\section{Enrico Ramirez-Ruiz}

University of California, Santa Cruz, USA

E-mail: enrico@ucolick.org

\section{Richard Strom}

Astron, Dwingeloo, Netherlands

University of Amsterdam, The Netherlands

E-mail: stromeastron.nl

We present results from two epochs global VLBI observations of XRF 080109/SN 2008D. With these sensitive global observations it is possible to give reasonable estimates to the speed of the radio ejecta. These early epoch data are crucial for the understanding of ejecta from type $\mathrm{Ib} / \mathrm{c}$ SNe.

The 9th European VLBI Network Symposium on The role of VLBI in the Golden Age for Radio Astronomy and EVN Users Meeting

September 23-26, 2008

Bologna, Italy

\footnotetext{
* Speaker.
} 


\section{Serendipitous discovery of XRF 080109/SN 2008D}

During Swift X-ray observations of SN 2007uy in NGC 2770 on January 9, a transient X-ray source was serendipitously discovered (Berger \& Soderberg 2008). This transient was hypothesized to be a weak X-ray flash (designated XRF 080109), XRFs being a sub-class of the enigmatic gamma-ray bursts (GRBs). The transient was also detected at optical (Deng \& Zhu 2008; Thoene et al. 2008) and radio (Soderberg 2008; van der Horst et al. 2008) wavelengths. The optical counterpart brightened rapidly (Valenti et al. 2008a) and spectroscopic observations suggested broad features possibly related to an emerging supernova (Soderberg et al. 2008a). It was classified as a type Ic supernova (Malesani et al. 2008; Valenti et al. 2008b) and designated SN 2008D. This source, which lies at a redshift of 0.007 or a distance of $30 \mathrm{Mpc}$, caused a multitude of observations across the whole electromagnetic spectrum.

XRFs are, similarly to a large fraction of the GRBs, caused by a relativistic jet emerging from the collapse of a massive star. A relativistic blast wave, ploughing its way through the ambient medium, accelerates electrons to extremely relativistic velocities, producing synchrotron radiation which is observed from radio to X-ray frequencies. In the case of nearby GRBs and XRFs the associated supernova emission is also detected, either by a late-time re-brightening in the optical light curves (Bloom et al. 1999; Galama et al. 2000) or by optical spectroscopy (Galama et al. 1998; Hjorth et al. 2003), the latter being possible for just four nearby GRBs/XRFs so far. While the optical emission can be completely dominated by the supernova, the radio emission is likely to come from the relativistic blast wave (Kaneko et al. 2007). The close distance to XRF 080109/SN 2008D provided an excellent opportunity to test this hypothesis by directly probing the angular size evolution of the ejecta by means of VLBI angular size measurements.

\section{Global VLBI observations}

We organized Target of Opportunity global VLBI observations on 6 February and 18 March 2008, 28 and 69 days after the discovery, respectively. The participating telescopes were Arecibo, Effelsberg, Jodrell Bank (MkII), Hartebeesthoek, Medicina, Noto, Onsala, Torun and Westerbork from the EVN, Hancock and St. Croix from the VLBA. The 8-hour observations (of which Arecibo could track the source only for about 1.5 hours) were carried out at $5 \mathrm{GHz}$ at 1024 Mbps using 2-bit sampling. The VLBA stations recorded at a rate of $512 \mathrm{Mbps}$ with 1-bit sampling, to obtain the same observing bandwidth. The synthesis array data from the WSRT were recorded parallel to the VLBI observations. The target was phase-referenced to the nearby calibrators J0911+3349 (0.8 deg.) and J0919+3324 (2 deg.), selected from the VLBA Calibrator list (http://www.vlba.nrao.edu/astro/calib/index.shtml). The phase-referencing cycle was 3:30-1:30 minutes for the target and the more nearby calibrator, including a 1 minute scan on the second calibrator as well in every other cycle.

\section{Determining the source size}

The data reduction was carried out in AIPS using standard techniques, and the calibrated data were exported to Difmap (Shepherd et al. 1994). The global VLBI images of XRF 080109/SN 2008D 

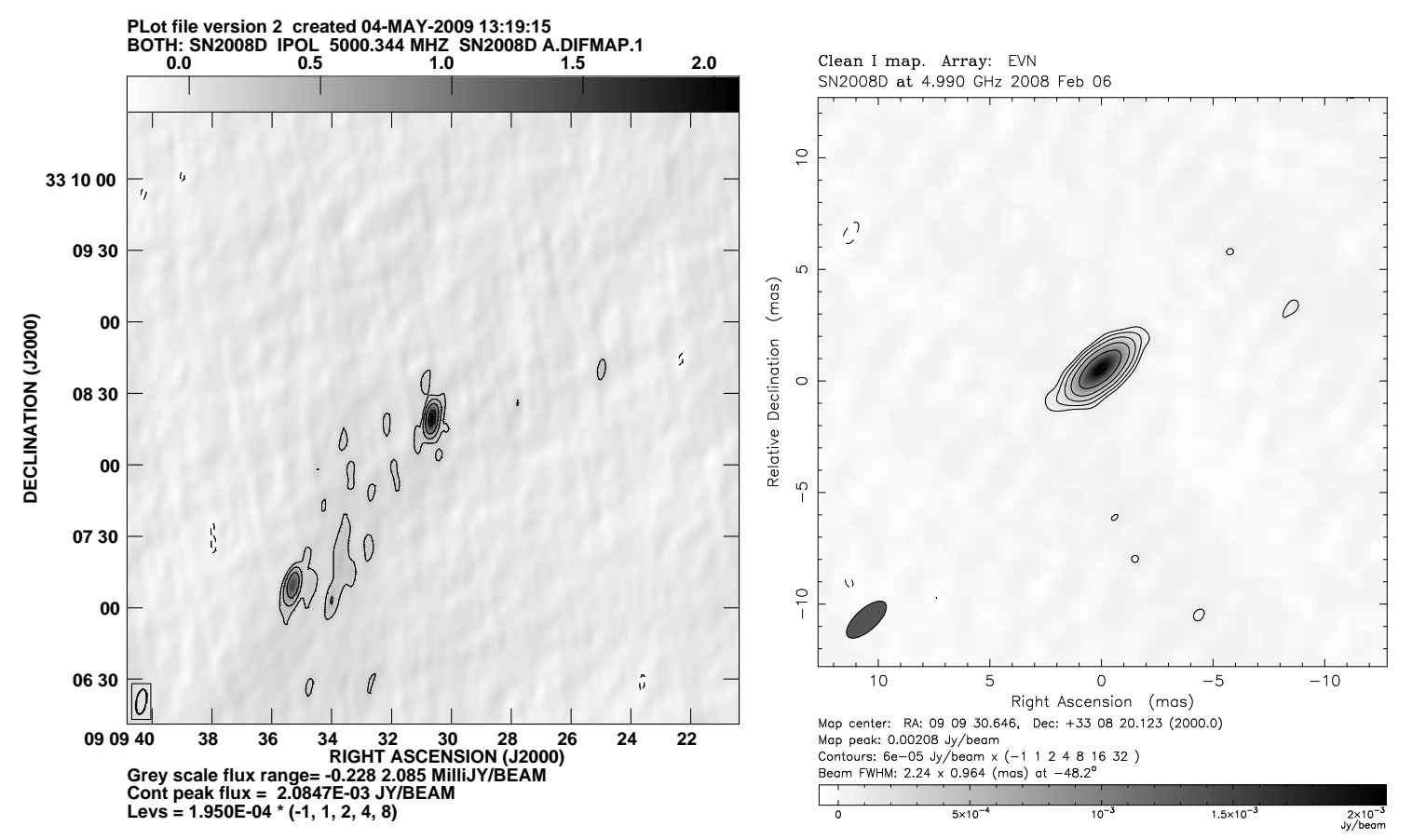

Figure 1: Left: Westerbork synthesis array map of XRF 080109/SN 2008D (centre of image) and SN 2007uy (lower left on image) in the nearby galaxy NGC 2770 . The beam was $10.6 \times 4.2$ arcseconds, PA=-10.3 degree. The data were recorded parallel to the VLBI observations. The main target source was about 2.1 mJy on 6 February 2008, and decreased to 0.7 mJy by 18 March 2008. Right: global VLBI image of XRF 080109/SN 2008D on 6 February 2008. The beam was $2.24 \times 0.96$ milliarcseconds, $P A=-48.2$ degree. Model-fitting results indicate that the source was marginally resolved.

appeared unresolved at both epochs. The observed peak brightnesses were $2.0 \mathrm{mJy} / \mathrm{beam}$ and $0.7 \mathrm{mJy} / \mathrm{beam}$ on 6 February and 18 March, respectively, with a far off-source image noise of 20 $\mu \mathrm{Jy} / \mathrm{beam}$. These values were consistent with the WSRT synthesis data measurements (Fig. 1). We carried out model-fitting of the $u v$-data in Difmap to give constraints on the apparent angular size. Because of the complex structure of the closer phase-reference source (J0911+3349), calibrating some of the less sensitive long baselines was challenging. Various phase-referencing calibration strategies were attempted, including (1) direct fringe-fitting on J0911+3349 and passing the solutions to the target, (2) first phase-referencing J0911+3349 to J0919+3324, and then the target to J0911+3349 via phase self-calibration, and (3) directly using J0919+3324 in fringe-fitting. Besides the phases, the amplitude solutions were varied as well by applying amplitude self-calibration on J0919+3324 and passing those to the target.

All these methods resulted in a similar value in source diameter (0.3-0.4 mas) when a circular Gaussian component was used as a model for the 6 February data. The data were not sufficient to constrain an elliptical Gaussian fit, especially because Arecibo could track the source only for a short time. Because of the very sensitive baselines in our dataset, and the relatively bright target source, we further attempted phase-only self-calibration using 1 hour solution intervals, to check the robustness of the fit. Either giving a point source starting model or a previously fitted circular 
Gaussian in self-calibration, the model-fitted source sizes afterwards were in agreement with the values mentioned above. This reassures us at least that the source is marginally resolved.

There is further uncertainty in the real brightness distribution of the source. A uniformly bright disk model would result in smaller a size ( $\sim 0.2$ mas), but that may be physically less appropriate, and produced a poorer fit. A ring model fit tends to result in a somewhat larger diameter for the target ( $\sim 0.5$ mas). Considering these uncertainties, we estimate the source to be $0.35 \pm 0.15$ mas. Work with the second epoch data is still ongoing. It has to be noted that because of the overwhelming sensitivity of Arecibo, which provides the longest of the most sensitive baselines, the results of our model-fitting could be strongly biased by possible phase errors for that particular telescope. This will need further, careful investigation.

\section{Discussion and Conclusions}

We give direct measurements to the size of radio ejecta from XRF 080109/SN 2008D at early epochs. Our results are in agreement with the measurements and upper limits derived from VLBA and HSA observations (Bietenholz, these proceedings). The source was only marginally resolved in our images, with a $3 \sigma$ upper limit of 0.5 mas at 28 days, resulting in an estimated upper limit on the apparent expansion velocity of 3.0c. The upper limit shows that if there was a relativistic jet, it was moderately relativistic. This result is consistent with the analysis of the radio light curves of this source (Soderberg et al. 2008b), which imply that the radio data are consistent with the stellar wind shocked by freely expanding non-relativistic ejecta as expected in core-collapse $\mathrm{SNe}$, and that the X-ray outburst is the shock break-out from a compact star embedded in a dense stellar wind. Further constraints on the expansion velocity of the ejecta will be obtained by determining (limits on) the proper motion of the source.

The importance of these early epoch observations is obvious: size measurements carried out years after the explosion would not constrain well the initial ejection speed, because of the significant deceleration on those timescales. Therefore, rapid-response VLBI measurements of Type Ib/c supernovae that are candidates for having a relativistic jet are crucial in pinning down the nature and physics of these stellar explosions.

\section{References}

[1] E. Berger \& A. M. Soderberg, ATel 1353 (2008)

[2] J. S. Bloom, S. R. Kulkarni, S. G. Djorgovski et al., Nature 401, 453 (1999)

[3] J. Deng \& Y. Zhu, GCN 7160 (2008)

[4] T. J. Galama, N. Tanvir, P. M. Vreeswijk et al., Astrophys. J. 536, 185 (2000)

[5] T. J. Galama, P. M. Vreeswijk, J. van Paradijs et al., Nature 395, 670 (1998)

[6] J. Hjorth, J. Sollerman, P. Moeller et al., Nature 423, 847 (2003)

[7] Y. Kaneko, E. Ramirez-Ruiz, J. Granot et al., Astrophys. J. 654, 385 (2007)

[8] D. Malesani, J. Hjorth, P. Jakobsson et al., GCN 7169 (2008)

[9] M. C. Shepherd, T. J. Pearson, G. B. Taylor, BAAS 26, 987 (1994) 

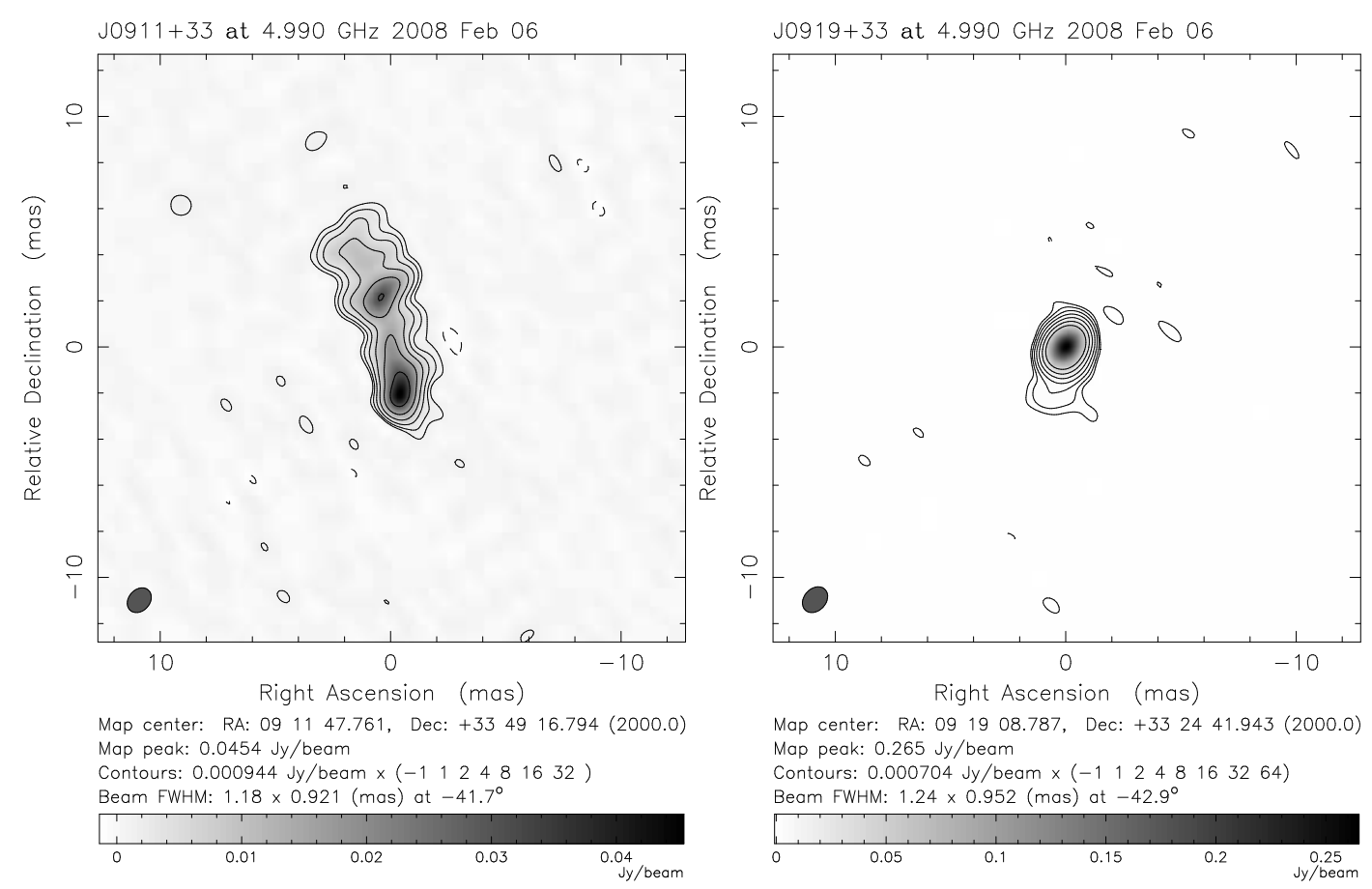

Figure 2: VLBI images of the nearby calibrators J0911+3349 (to the left) and J0919+3324 (to the right).

[10] A. M. Soderberg, GCN 7178 (2008)

[11] A. M. Soderberg, E. Berger, D. Fox et al., GCN 7165 (2008a)

[12] A. M. Soderberg, E. Berger, K. L. Page et al., Nature 453, 469 (2008b)

[13] C. C. Thoene, D. Malesani, J. Hjorth et al., GCN 7161 (2008)

[14] S. Valenti, M. Turatto, H. Navasardyan et al., GCN 7163 (2008)

[15] S. Valenti, D. Fugazza, E. Maiorano et al., GCN 7171 (2008)

[16] A. J. van der Horst, C. Kouveliotou, R. .A. M. J. Wijers \& A. Kamble, GCN 7190 (2008) 